\title{
Phase transitions in LaFeAsO: Structural, magnetic, elastic, and transport properties, heat capacity and Mössbauer spectra
}

\author{
Michael A. McGuire, Andrew D. Christianson, Athena S. Sefat, Brian C. Sales, Mark D. Lumsden, Rongying Jin, \\ E. Andrew Payzant, and David Mandrus \\ Oak Ridge National Laboratory, Oak Ridge, Tennessee 37831, USA \\ Yanbing Luan and Veerle Keppens \\ Department of Materials Science and Engineering, The University of Tennessee, Knoxville, Tennessee 37996-2200, USA
}

Vijayalaksmi Varadarajan and Joseph W. Brill

Department of Physics and Astronomy, University of Kentucky, Lexington, Kentucky 40506-0055, USA

Raphaël P. Hermann

Institut für Festkörperforschung, Forschungszentrum Jülich GmbH, D-52425 Jülich, Germany

and Department of Physics, B5, Université de Liège, B-4000 Sart-Tilman, Belgium

Moulay T. Sougrati and Fernande Grandjean

Department of Physics, B5, Université de Liège, B-4000 Sart-Tilman, Belgium

Gary J. Long

Department of Chemistry, Missouri University of Science and Technology, Rolla, Missouri 65409-0010, USA

(Received 24 June 2008; published 24 September 2008)

\begin{abstract}
We present results from a detailed experimental investigation of $\mathrm{LaFeAsO}$, the parent material in the series of "FeAs" based oxypnictide superconductors. Upon cooling, this material undergoes a tetragonalorthorhombic crystallographic phase transition at $\sim 160 \mathrm{~K}$ followed closely by an antiferromagnetic ordering near $145 \mathrm{~K}$. Analysis of these phase transitions using temperature dependent powder $\mathrm{x}$-ray and neutrondiffraction measurements is presented. A magnetic moment of $\sim 0.35 \mu_{B}$ per iron is derived from Mössbauer spectra in the low-temperature phase. Evidence of the structural transition is observed at temperatures well above the transition temperature (up to near $200 \mathrm{~K}$ ) in the diffraction data as well as the polycrystalline elastic moduli probed by resonant ultrasound spectroscopy measurements. The effects of the two phase transitions on the transport properties (resistivity, thermal conductivity, Seebeck coefficient, and Hall coefficient), heat capacity, and magnetization of $\mathrm{LaFeAsO}$ are also reported, including a dramatic increase in the magnitude of the Hall coefficient below $160 \mathrm{~K}$. The results suggest that the structural distortion leads to a localization of carriers on $\mathrm{Fe}$, producing small local magnetic moments which subsequently order antiferromagnetically upon further cooling. Evidence of strong electron-phonon interactions in the high-temperature tetragonal phase is also observed.
\end{abstract}

DOI: 10.1103/PhysRevB.78.094517

PACS number(s): $74.10 .+\mathrm{v}$

\section{INTRODUCTION}

The family of lanthanide iron oxypnictides crystallizing in the $\mathrm{ZrCuSiAs}$ structure type has received extensive attention in the recent condensed-matter literature. Many of these compounds have been known for almost a decade, ${ }^{1,2}$ and superconductivity below $\sim 7 \mathrm{~K}$ was reported in fluorine doped LaMPO (M=Fe, Ni) in 2006 and 2007. ${ }^{3-5}$ However, the discovery of superconductivity at $28 \mathrm{~K}$ in fluorine doped $\mathrm{LaFeAsO},{ }^{6}$ and the subsequent reports of transition temperatures greater than $50 \mathrm{~K}$ in some of the related rare-earth materials have generated great interest and a flurry of recent experimental and theoretical activity, generating preprints daily at arxiv.org/archive/cond-mat and numerous publications in just the first few months. ${ }^{7-27}$ The superconductivity in these materials appears to be unconventional, ${ }^{7,8,28}$ and much careful work will be required to elucidate the interesting physics in this family of compounds. In the arsenic con- taining materials doping is required to produce the superconducting state, and much work is duly focused on the doping behavior of these compounds. However, study of the undoped materials is also important in developing an understanding of the superconductivity.

The undoped material $\mathrm{LaFeAsO}$ has been reported to undergo a spin-density wave (SDW) transition near $150 \mathrm{~K}, 6,29$ based on specific heat, resistivity, and reflectivity measurements. A structural phase transition has also been reported in this material at temperatures just above the magnetic transition. ${ }^{12,30}$ Upon doping with fluorine the SDW is suppressed and superconductivity emerges. ${ }^{6,8,29}$ Careful characterization of the behavior of $\mathrm{LaFeAsO}$ and other materials in this family is important in understanding the underlying physics responsible for these behaviors. Here we report the results of our experimental investigation of $\mathrm{LaFeAsO}$. We present structural analysis through the crystallographic phase transition from powder x-ray diffraction (PXRD) and inves- 
tigation of the magnetic transition using neutron powder diffraction (NPD) along with magnetization and Mössbauer spectral measurements. Signatures of these phase transitions are observed in ac-calorimetry heat capacity and resonant ultrasound spectroscopy (RUS) measurements. The effects of the structural and magnetic transitions on the transport properties of $\mathrm{LaFeAsO}$ (electrical resistivity, magnetoresistance, Hall effect, Seebeck effect, and thermal conductivity) are also examined.

\section{EXPERIMENTAL DETAILS}

\section{A. Sample synthesis}

We tried several methods for synthesizing high quality samples of LaFeAsO. Starting materials were from Alfa Aesar and included $\mathrm{La}(99.9 \%), \mathrm{La}_{2} \mathrm{O}_{3} \quad(99.99 \%), \quad \mathrm{Fe}$ $(99.998 \%), \mathrm{Fe}_{2} \mathrm{O}_{3}(99.998 \%)$, and As $(99.999 \%)$. The binaries LaAs and FeAs were synthesized for use as precursors by heating slowly over the course of several days stoichiometric mixtures of the elements in sealed, evacuated silica tubes to $950^{\circ} \mathrm{C}$ for LaAs and $1050^{\circ} \mathrm{C}$ for FeAs, and soaking for 12-24 h. Different synthesis routes will be distinguished by capital letters A-D in the discussion below.

Synthesis route A used a finely ground stoichiometric mixture of LaAs, Fe, and $\mathrm{Fe}_{2} \mathrm{O}_{3}$ pressed into a pellet, wrapped in Ta foil and heated in a sealed silica tube partially backfilled with ultra-high-purity Ar and with a small piece of $\mathrm{Zr}$ foil, and heated at $1200^{\circ} \mathrm{C}$ for about one day. Powder $\mathrm{X}$-ray diffraction analysis showed the product to be nearly single phase $\mathrm{LaFeAsO}$. The only other phases observed by PXRD were $\mathrm{Fe}$ and $\mathrm{La}_{2} \mathrm{O}_{3}$, while neutron-diffraction experiments detected $\mathrm{Fe}_{2} \mathrm{As}$. Rietveld analysis ${ }^{31-33}$ indicates that the amount of each of these impurities was less than $5 \%$. It is likely that the iron containing impurities result from the reaction of the $\mathrm{Zr}$ foil with As vapor in the tube, reducing FeAs (a typical impurity phase when no metal foils are present) to $\mathrm{Fe}_{2} \mathrm{As}$ and $\mathrm{Fe}$.

Synthesis route B is identical to A except no metal foils were used. This typically gives a product with significant $(\sim 10 \%) \quad \mathrm{La}_{2} \mathrm{O}_{3}$ and FeAs impurities as determined by neutron-diffraction and Mössbauer spectroscopy, but no Fe nor $\mathrm{Fe}_{2} \mathrm{As}$.

Synthesis route $\mathrm{C}$ involves reacting the product of route $\mathrm{B}$ with a small excess of La powder (a few percent). We found that this helps to further react the $\mathrm{La}_{2} \mathrm{O}_{3}$ and $\mathrm{FeAs}$ impurities left from route $\mathrm{B}$ and can produce PXRD pure material. Occasionally small amounts of $\mathrm{LaAs}$ and/or $\mathrm{La}_{2} \mathrm{O}_{3}$ are observed in the products from route $\mathrm{C}$.

Route D used a finely ground stoichiometric mixture of FeAs, $\mathrm{La}_{2} \mathrm{O}_{3}$, and La pressed into a pellet, sealed in a silica tube partially backfilled with ultra-high-purity Ar, and heated at $1200^{\circ} \mathrm{C}$ for $30-36 \mathrm{~h}$. This method produced purer samples, sometimes with no impurities observable by PXRD.

A single sample prepared by route A was used for neutron diffraction, Hall effect, resistivity, magnetoresistance, thermal conductivity, Seebeck coefficient, and heat-capacity measurements reported below. The presence of elemental Fe precluded magnetic characterization of this sample; the material was strongly magnetic at room temperature. Magneti- zation measurements reported below were carried out on a PXRD pure sample obtained via route C. Material produced by route B was used for Mössbauer spectroscopy and elastic constant measurements. The temperature dependent PXRD data presented here were collected using a sample made by route $\mathrm{D}$, and are in good agreement with similar measurements on the sample used for transport, NPD, and heatcapacity measurements (route A).

\section{B. Characterization techniques}

Temperature dependent PXRD data were collected using an Anton Parr TTK450 low-temperature stage on a PANalytical X'pert Pro MPD with an X'celerator position sensitive detector and using $\mathrm{Cu} K \alpha$ radiation. The PXRD sample was mixed with copper powder in a small amount of vacuum grease to calibrate the sample temperature for each scan using the refined lattice constant of $\mathrm{Cu}$, and to increase the thermal contact between the sample and the holder. Neutron diffraction measurements were performed at the High Flux Isotope Reactor (HFIR) on $\sim 1.5 \mathrm{~g}$ samples of $\mathrm{LaFeAsO}$ and $\mathrm{LaFeAsO}_{0.89} \mathrm{~F}_{0.11}$ on the HB1A triple-axis spectrometer with horizontal collimations of 48-40-40-68 using a highly filtered beam $\left(\lambda / 2 \sim 10^{-4} \lambda\right)$ with an incident energy of 14.64 meV. Preliminary measurements were also performed on the WAND diffractometer and the HB3 triple-axis spectrometer. Data were collected over a range of scattering angles from $5^{\circ}$ to $130^{\circ}$ at several temperatures.

Transport measurements were performed using a Quantum Design Physical Property Measurement System (PPMS). Silver epoxy (Epotek H20E) was used for electrical and thermal contacts. Hall effect in fields up to $6 \mathrm{~T}$ and electrical resistivity measurements were made using platinum wire leads. Gold-coated copper leads were used for thermal conductivity and Seebeck coefficient measurements.

Heat capacity was determined by ac-calorimetry measurements made on a $3.18 \mathrm{mg}$ polycrystalline sample, using 4.5 $\mathrm{Hz}$ chopped white light as a heating source. ${ }^{34}$ The technique only yields relative values of the heat capacity, so the results were normalized at $127 \mathrm{~K}$ to lower resolution specific-heat data taken with the PPMS. The data were then corrected for the addendum heat capacity (thermocouple wire plus glue), which was $\sim 2.5 \%$ of the sample's in the temperature region measured.

The elastic moduli were measured using resonant ultrasound spectroscopy (RUS) and a custom made probe in a Quantum Design PPMS cryostat. ${ }^{35}$ Stanford Research synthesized function generator and model SR 844 RF lock-in amplifier were used to excite the sample and collect the data.

The iron-57 Mössbauer spectra were recorded between 4.2 and $295 \mathrm{~K}$ on a constant acceleration spectrometer which utilized a rhodium matrix cobalt-57 source and was calibrated at $295 \mathrm{~K}$ with $\alpha$-iron powder. The isomer shifts are relative to room-temperature $\alpha$ iron. The accuracy of the temperature is better than $\pm 1 \%$.

\section{RESULTS AND DISCUSSION}

\section{A. X-ray diffraction: Structural phase transition}

It has been reported that $\mathrm{LaFeAsO}$ undergoes a structural phase transition from tetragonal $(P 4 / \mathrm{nmm}$, space-group 


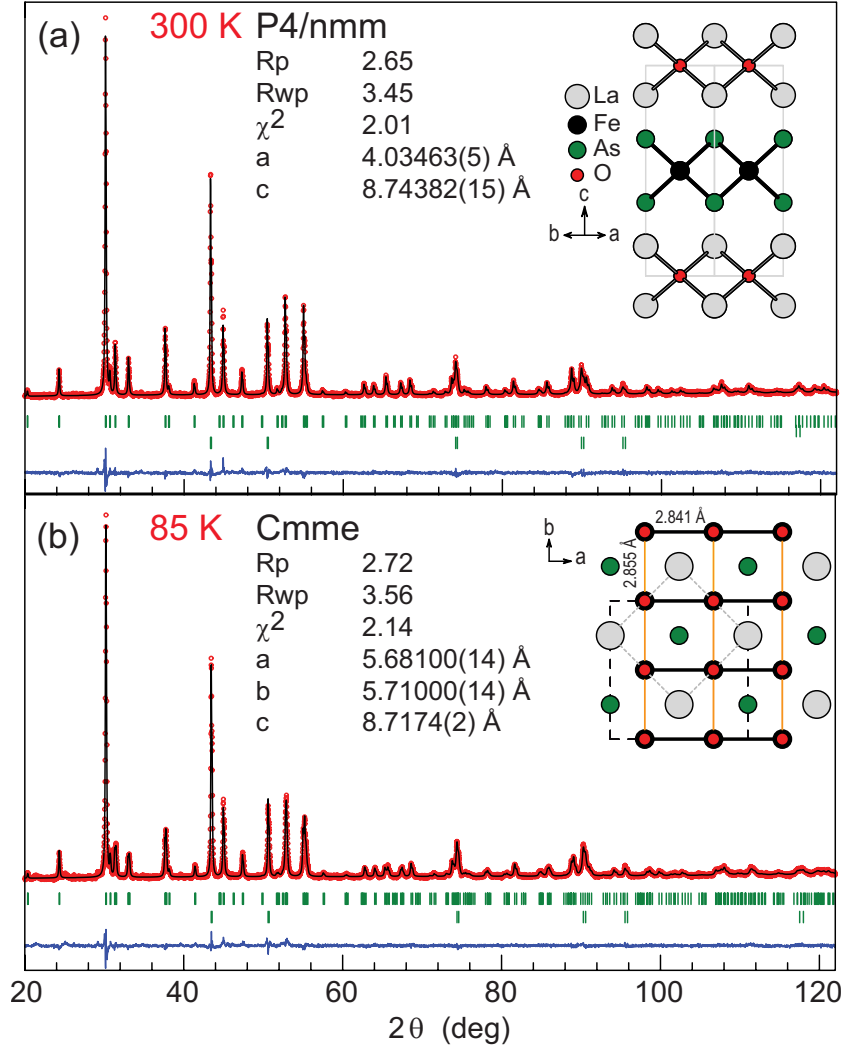

FIG. 1. (Color online) Rietveld refinement results for $\mathrm{LaFeAsO}$ at (a) $300 \mathrm{~K}$ and (b) $85 \mathrm{~K}$. The upper sets of ticks mark the location of Bragg peaks from $\mathrm{LaFeAsO}$, while the lower ticks locate Bragg peaks from the $\mathrm{Cu}$ internal standard. The inset in (a) shows the tetragonal structure viewed along ( $\left.\begin{array}{lll}1 & 1 & 0\end{array}\right)$ emphasizing the FeAs layer in this material. The gray line outlines the tetragonal unit cell. The inset in (b) shows the orthorhombic structure viewed along $(00$ $1)$, emphasizing the distortion which occurs in the $a b$ plane upon cooling through the tetragonal to orthorhombic phase transition. The dashed black lines outline the $c$-centered orthorhombic unit cell, while the dotted gray lines show the primitive unit cell (see text).

number 129) to orthorhombic (Cmme, space group formerly known as Cmma, number 67) upon cooling at about 155 K. ${ }^{12,30}$ Figure 1 shows the Rietveld refinement results of powder X-ray diffraction data collected at 300 and at $85 \mathrm{~K}$ (sample made by synthesis route D). Good fits to the reported structural models are observed. The inset of Fig. 1(a) shows the high-temperature tetragonal structure viewed along the $\left(\begin{array}{lll}1 & 1 & 0\end{array}\right)$ direction. The layered nature of the structure emphasized in Fig. 1(a) persists into the lowtemperature orthorhombic structure. The inset of Fig. 1(b) shows a slab containing one layer of each atom type viewed perpendicular to the layers (along the $c$ axis). Upon cooling through the phase transition the square nets of atoms present in the tetragonal structure distort into rectangular nets of $\mathrm{Fe}$ and of $\mathrm{O}$, and into centered rectangular nets of $\mathrm{La}$ and of As. The two Fe-Fe distances in the low-temperature phase are labeled in Fig. 1(b). For comparison, the Fe-Fe distance in the square nets at $300 \mathrm{~K}$ is $2.853 \AA$.

The structures have been described in detail elsewhere. ${ }^{12,30}$ However, some confusion exists regarding the symmetry of the low-temperature structure. It has been reported in both primitive monoclinic and $c$-centered orthorhombic space groups. In the monoclinic description the reported lattice is metrically orthorhombic and atoms are very close to positions which would give orthorhombic symmetry to the crystal as well. The orthorhombic description is probably correct, and no indication of deviation from orthorhombic symmetry is observed in our PXRD data.

To identify the temperature at which the tetragonalorthorhombic structural transition occurs, temperature dependent diffraction data were refined in both models. Figure 2(a) compares the goodness of fit $\left(\chi^{2}\right)$ obtained from each refinement, and shows that the orthorhombic model gives a better fit at $180 \mathrm{~K}$ and below. This suggests that the structural transition begins at temperatures significantly higher than previously reported. In addition, we compare in Fig. 2(a) the width of two Bragg peaks, one which is split by the structural distortion $\left(\begin{array}{lll}1 & 1 & 0\end{array}\right)$ and one which is not $\left(\begin{array}{lll}2 & 0 & 0\end{array}\right)$. We note that indices refer to the tetragonal structure. The results show that while the $\left(\begin{array}{lll}2 & 0 & 0\end{array}\right)$ peak width is unchanged upon cooling, the $\left(\begin{array}{lll}1 & 1 & 0\end{array}\right)$ peak begins to broaden significantly at about 180 $\mathrm{K}$.

Figure 2(b) shows the temperature dependence of the refined lattice constants. Determining the temperature at which to switch between the Cmme and P4/ $\mathrm{nmm}$ models is not straightforward. We chose to show data in the Cmme model when the difference between the refined values of the $a$ and $b$ axes is greater than the error associated with these parameters. Based on this criterion, the Cmme model was used at $200 \mathrm{~K}$ and below. Above this temperature refinement in this model is in fact unstable and $P 4 / \mathrm{nmm}$ was used. The refined values of the lattice constants $a$ and $b$ show a gradual divergence below $200 \mathrm{~K}$ followed by a more rapid divergence at lower temperatures. The temperature dependence of the lattice constant $c$ is shown in the inset of Fig. 2(b), and undergoes a change in slope through the structural transition.

The above observations suggest that the structural distortion in LaFeAsO occurs over a wide temperature range, but includes a "sharp" anomaly as well. To derive a transition temperature $\mathrm{T}_{T-O}$ from the structural data, we plot the difference between the lattice constants $a$ and $b$ in Fig. 2(c). These data show an abrupt slope change at about $160 \mathrm{~K}$ which we identify as $\mathrm{T}_{T-O}$. We note, however, that the onset temperature of this distortion $(180-200 \mathrm{~K})$ is significantly higher than $\mathrm{T}_{T-O}$. We believe this extension of the lattice distortion to temperatures well above the "transition temperature" is observable in the data shown in previous reports which interpreted the results as a single sharp transition. ${ }^{12,30}$ Perhaps the two-dimensional nature of the crystal and electronic structures leads to enhanced fluctuations above the transition temperature.

\section{B. Neutron diffraction: Magnetic phase transition}

Neutron diffraction results reveal a magnetic transition somewhat below the temperature at which the structural transition occurs. Figure 3(a) shows neutron-diffraction data for both $\mathrm{LaFeAsO}$ (synthesis route $\mathrm{A}$ ) and superconducting $\mathrm{LaFeAsO}_{0.89} \mathrm{~F}_{0.11}$ (Ref. 8) at 70 and $200 \mathrm{~K}$ from $32^{\circ}$ to $36^{\circ}$ in 

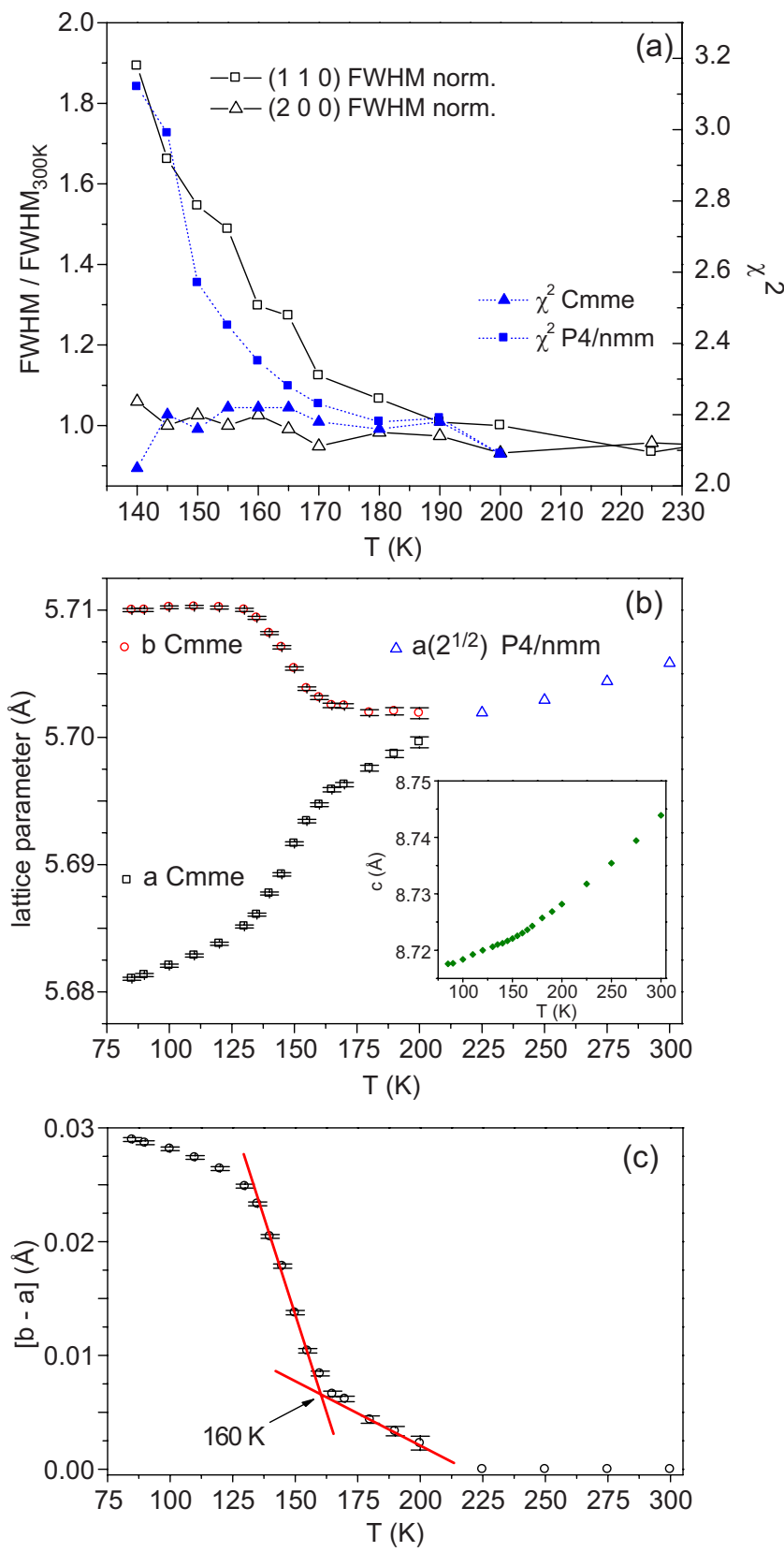

FIG. 2. (Color online) Results of temperature dependent PXRD analysis of LaFeAsO showing the continuous nature of the crystallographic phase transition. (a) Solid symbols show $\chi^{2}$ from Rietveld refinement using the orthorhombic low-temperature model and tetragonal high-temperature model. Open symbols show the normalized width of two Bragg peaks labeled by their tetragonal indices. The $\left(\begin{array}{lll}1 & 1 & 0\end{array}\right)$ peak is expected to be split into two peaks by the

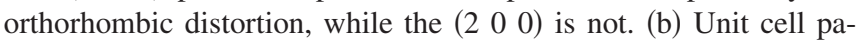
rameters determined by Rietveld refinement. For comparison the $a$ lattice parameter in the tetragonal structure has been multiplied by $\sqrt{2}$. Error bars are not shown on some data points for which the error is smaller than the size of the data point symbol. (c) The difference between $b$ and $a$ in the orthorhombic model, linearly coupled to the order parameter for the structural phase transition. We define the transition temperature for the tetragonal to orthorhombic transition as the kink near $160 \mathrm{~K}$, but note that the structure is evolving continuously both above and below this temperature.

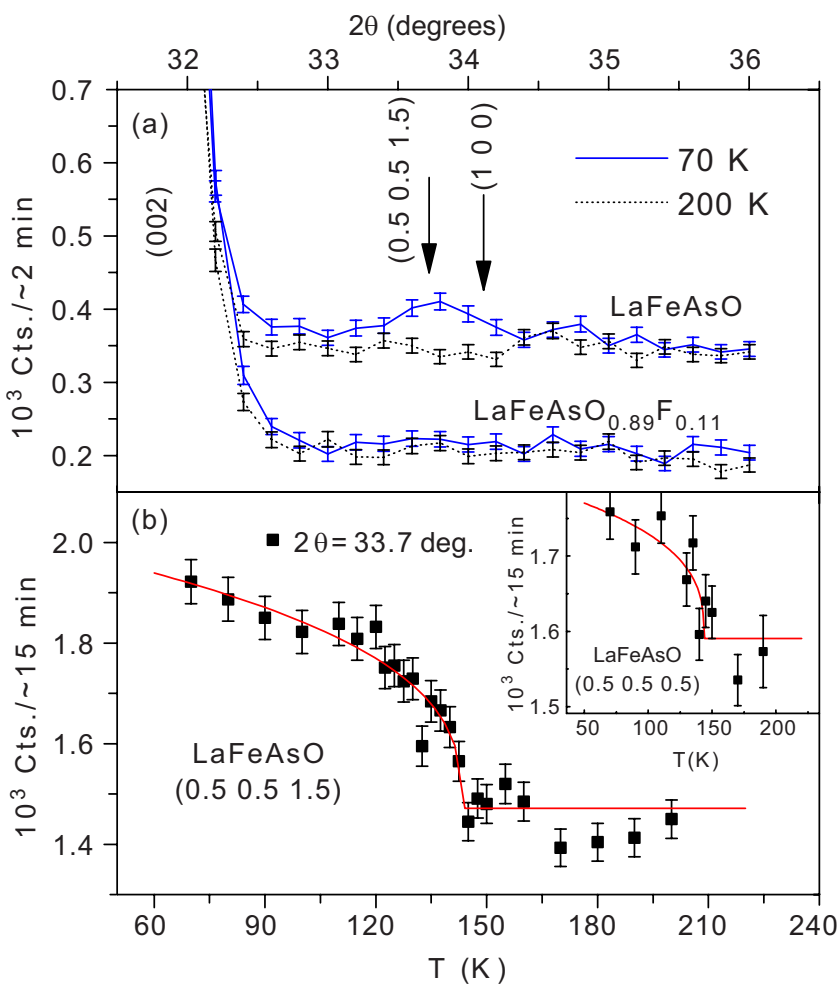

FIG. 3. (Color online) (a) Neutron diffraction data for LaFeAsO and $\mathrm{LaFeAsO}_{0.89} \mathrm{~F}_{0.11}$ at 70 and $200 \mathrm{~K}$. Arrows indicate the angular position of the $\left(\begin{array}{lll}1 & 0 & 0\end{array}\right)$ and $\left(\begin{array}{lll}0.5 & 0.5 & 1.5\end{array}\right)$ wave vectors. For clarity, the data for $\mathrm{LaFeAsO}$ have been displaced by 150 counts. (b) The temperature dependence of the $(0.50 .51 .5)$ and $(0.50 .50 .5)$ (inset) positions. The wave vectors are labeled using the tetragonal setting. The lines are a guide to the eye.

scattering angle. These data indicate the presence of additional scattering at $33.7^{\circ}$ at $70 \mathrm{~K}$ that is not present at $200 \mathrm{~K}$. Figure $3(\mathrm{~b})$ shows the temperature dependence of the scattering at $33.7^{\circ}$. These data show that the additional lowtemperature intensity in $\mathrm{LaFeAsO}$ appears below $145 \mathrm{~K}$. Polarized measurements (not shown) indicate that this scattering is magnetic in nature. The same angular range was explored in $\mathrm{LaFeAsO}_{0.89} \mathrm{~F}_{0.11}$. Although the counting time was doubled to 4 min per point, [normalized in Fig. 3(a) to 2 min per point for the purposes of comparison] there is no discernable difference between the data at 70 and 200 K. Full refinements of the neutron data indicate similar impurity phases in both samples and, hence, the extra intensity is intrinsic to $\mathrm{LaFeAsO}$. For reference, the strong rise in intensity below $32.5^{\circ}$ corresponds to the $\left(\begin{array}{ll}0 & 0\end{array}\right)$ structural Bragg peak.

The arrows in Fig. 3(a) show the position of two plausible wave vectors (labeled in the tetragonal setting) to describe the additional intensity at $33.7^{\circ}$ in $\mathrm{LaFeAsO}$. The position of ( $\left.\begin{array}{lll}1 & 0 & 0\end{array}\right)$ is significantly far from the observed peak in the intensity to rule it out as the wave vector. However, the data are strongly consistent with a wave vector of $(0.50 .51 .5)$. To check the indexing of this peak, we searched for extra intensity at $(0.50 .50 .5)$. The intensity observed at this location is very weak but careful measurement of the temperature dependence shown in the inset of Fig. 3(b) indicates an increase below $145 \mathrm{~K}$ in a manner consistent with the $33.7^{\circ}$ 


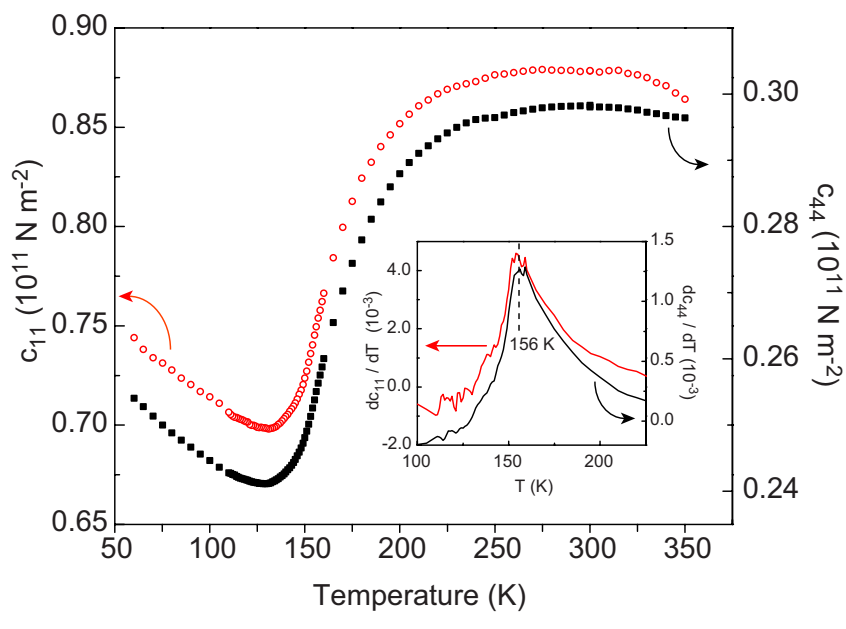

FIG. 4. (Color online) Results of resonant ultrasound spectroscopy studies showing the effects of the crystallographic phase transition on the polycrystalline elastic moduli $\mathrm{c}_{11}$ and $\mathrm{c}_{44}$ of LaFeAsO. A gradual softening begins above $200 \mathrm{~K}$, well above the structural transition, and extends down to $140 \mathrm{~K}$. A relatively sharp peak in the derivative (inset) occurs near $\mathrm{T}_{T-O}$.

reflection. The observation of these two reflections provides strong evidence that the true ordering wave vector in

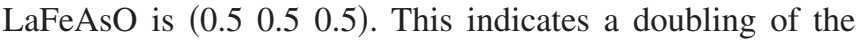
conventional unit cell along both $a$ axes and along the $c$ axis, and is also consistent with the $\sqrt{2} \times \sqrt{2}$ unit cell suggested by Dong et al., ${ }^{29}$ and is in good agreement with the magnetic structure derived from other recent neutron-diffraction experiments on $\mathrm{LaFeAsO} .{ }^{12}$ Evidence of this antiferromagnetic ordering is also observed in the magnetization data discussed below.

\section{Elastic constants}

Resonant ultrasound spectroscopy (RUS) measurements were performed to investigate the elastic properties of LaFeAsO. This technique can be a very sensitive and powerful probe for studying phase transitions, in particular structural transitions in which strain is the order parameter. Figure 4 shows the temperature dependence of the polycrystalline elastic moduli $\mathrm{c}_{11}$ and $\mathrm{c}_{44}$. A remarkably gradual softening is seen to extend up to above $200 \mathrm{~K}$. Data are shown for a sample made by synthesis route B; similar behavior was observed in material from synthesis route A. The temperature derivative of the elastic moduli are shown in the inset of Fig. 4. The sharp cusp in $d c_{i j} / d T$ occurs at $156 \mathrm{~K}$ and is identified as the structural transition temperature determined by this experimental probe. This agrees well with $\mathrm{T}_{T-O}$ determined by the structural analysis presented above. The extension of the elastic softening to well above $\mathrm{T}_{T-O}$ is consistent with the PXRD data presented above and is further evidence of the gradual nature of the crystallographic phase change in this material. We also note that no significant thermal hysteresis or magnetic field effect on the elastic properties was observed.

\section{Heat capacity}

The measured heat capacity of LaFeAsO (synthesis route A) in the vicinity of the structural and magnetic transitions is

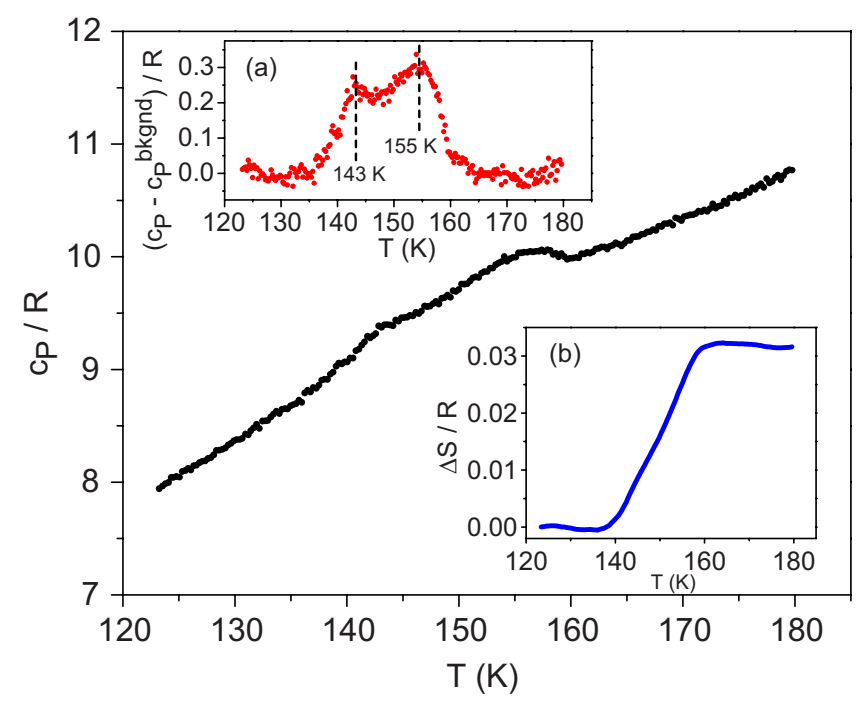

FIG. 5. (Color online) The measured heat capacity of LaFeAsO (in units of R/f.u.) which displays broad anomalies at both phase transitions. Inset (a) shows the baseline subtracted data which clearly show two broad peaks centered near the magnetic and structural transition temperatures. Inset (b) shows the integrated entropy associated with the two anomalies.

shown in Fig. 5. Two broad overlapping anomalies are observed. We note that two heat-capacity anomalies were also observed in sample used for PXRD analysis, produced by route D. Baseline subtracted data are shown in Fig. 5, inset (a). The background was estimated by a polynomial fit to the data above and below the transition region. The subtracted data clearly show two peaks, one associated with the structural transition centered at $155 \mathrm{~K}$, and one due to the magnetic transition centered at $143 \mathrm{~K}$. The coincidence of these two peaks with the structural and magnetic phase-transition temperatures strongly suggest that these are indeed separate anomalies, and not a single transition smeared by, for example, inhomogeneities. No hysteresis was observed in repeated measurements, suggesting that the phase transitions are second order or perhaps weakly first order. The entropy change determined by integration of the subtracted heatcapacity data is shown in Fig. 5, inset (b). A total entropy of $0.032 \mathrm{R}\left(0.27 \mathrm{~J} \mathrm{~K}^{-1} \mathrm{~mol} \mathrm{f.u}^{-1}\right)$ is determined by the integration. If the total change in entropy is considered purely electronic then $\Delta \gamma \sim 1.7 \mathrm{~mJ} \mathrm{~mol}^{-1} \mathrm{~K}^{-2}$.

\section{E. Transport properties}

The transport properties reported here were all measured on a single sample produced using synthesis route A. Figure 6(a) shows the measured electrical resistivity of $\mathrm{LaFeAsO}$, which agrees well with previous reports. ${ }^{36}$ At room temperature $\rho$ has a value of $4 \mathrm{~m} \Omega \mathrm{cm}$ and decreases upon cooling. This is typical of a low carrier-concentration metal or heavily doped semiconductor. The upturn in $\rho$ on cooling below about $200 \mathrm{~K}$ is likely due to increased charge-carrier scattering by lattice fluctuations related to the onset of the impending structural transition. The electrical resistivity reaches a local maximum at $165 \mathrm{~K}$ and drops rapidly below this temperature. 

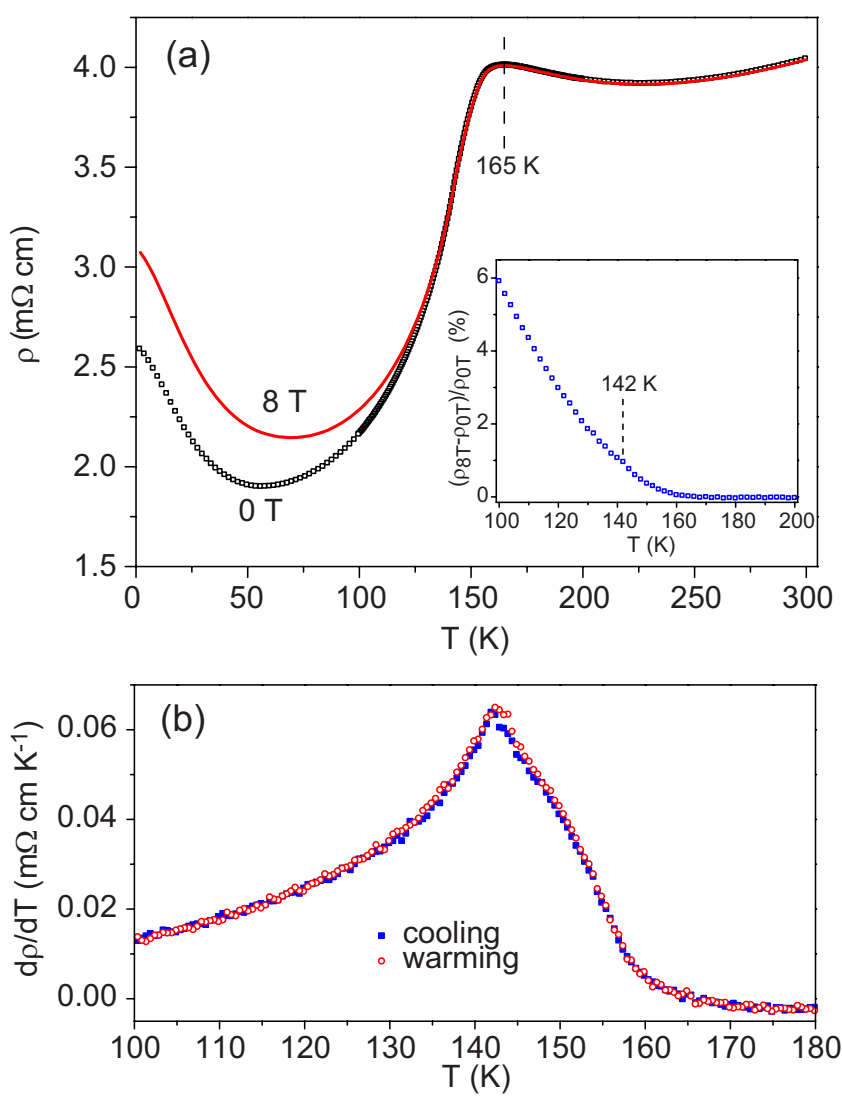

FIG. 6. (Color online) Effects of the phase transitions on the electrical transport of LaFeAsO. (a) The temperature dependence of the electrical resistivity with no applied magnetic field and with an applied field of $8 \mathrm{~T}$. The inset in (a) shows the magnetoresistance calculated from the resistivity data. (b) The temperature derivative of the measured resistivity on cooling and warming illustrating the absence of thermal hysteresis. The effect of the structural transition at $160 \mathrm{~K}$ is shown. The peak in $d \rho / d T$ is near the magnetic transition temperature at $143 \mathrm{~K}$.

The temperature derivative of the measured resistivity data is shown in Fig. 6(b). Below about $160 \mathrm{~K}$ a sharp increase in $d \rho / d T$ is observed. The maximum occurs at $143 \mathrm{~K}$, near the magnetic transition temperature. No thermal hysteresis is observed in these data, suggesting that neither of the phase transitions are strongly first order. Since the resistivity anomaly begins near $\mathrm{T}_{T-O}=160 \mathrm{~K}$ and this is the temperature below which significant magnetoresistance appears [Fig. 6(a) inset], we believe that structural phase transition in $\mathrm{LaFeAsO}$ is primarily responsible for the dramatic changes in transport properties. The magnetic transition occurs near the peak in $d \rho / d T$, and may be responsible for the weak anomaly observed at $142 \mathrm{~K}$ in the magnetoresistance [Fig. 6(a), inset].

Results of Hall-effect measurements are shown in Fig. 7. Near room temperature the Hall coefficient is negative and nearly temperature independent, indicating conduction by electrons with an inferred concentration $n=3 \times 10^{21} \mathrm{~cm}^{-3}$. We note that the presence of multiple bands at the Fermi level complicates the interpretation of the Hall-coefficient data. In a simple model with one-electron band and one hole band the inferred value of $n$ gives an upper bound on the
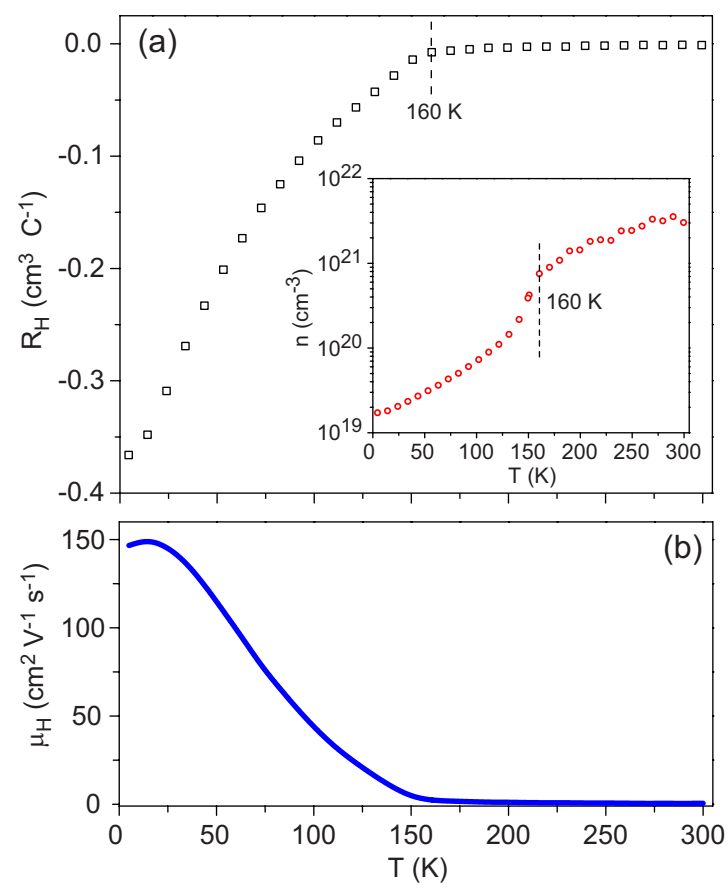

FIG. 7. (Color online) Results of Hall-effect measurements on $\mathrm{LaFeAsO}$ showing the remarkable decrease in inferred carrier concentration and high mobility at low temperatures. (a) The measured Hall coefficient and inferred carrier concentration (inset). (b) The Hall mobility calculated from the carrier concentration in (a) and the electrical resistivity (Fig. 6).

concentration of the dominant charge carriers. Upon cooling below $160 \mathrm{~K}$ the Hall coefficient decreases rapidly, and $n$ drops by an order of magnitude between 160 and $100 \mathrm{~K}$ [Fig. $7(\mathrm{a})$, inset]. This suggests that many of the charge carriers present in the high-temperature phase are localized at $\mathrm{T}_{T-O}$ due to the structural transition. This is possibly related to the local-moment formation and subsequent magnetic ordering observed below $\mathrm{T}_{T-O}$. It is interesting that this order of magnitude decrease in $n$ coincides with a factor of 2 decrease in $\rho$. This suggests a large change in carrier mobility. The Hall mobility $\mu_{H}=R_{H} \rho^{-1}$ is plotted in Fig. 7(b). The mobility increases by a factor of 300 between room temperature and 5 $\mathrm{K}$, and reaches the remarkably high value of $150 \mathrm{~cm}^{2} \mathrm{~V}^{-1} \mathrm{~s}^{-1}$ in this polycrystalline material.

The measured thermal conductivity $\kappa$ and Seebeck coefficient $S$ of LaFeAsO are shown in Fig. 8. The thermal conductivity increases abruptly below about $155 \mathrm{~K}$, but otherwise follows the behavior of typical crystalline materials. The increase in $\kappa$ cannot be attributed to the change in electronic thermal conduction as the thermal conductivity in this low carrier-concentration material is dominated by phonons. Thus the observed behavior must be attributed to an increase in the thermal conductivity of the lattice, and related to the tetragonal-orthorhombic crystallographic transition that occurs at $160 \mathrm{~K}$ (Fig. 8, inset a). This could be due to a decrease in electron-phonon scattering below the transition, which would suggest strong coupling between the charge carriers and the lattice vibrations in the tetragonal phase of $\mathrm{LaFeAsO}$ through bond-length fluctuations. This is also consistent with the rapid increase in the carrier mobility pre- 


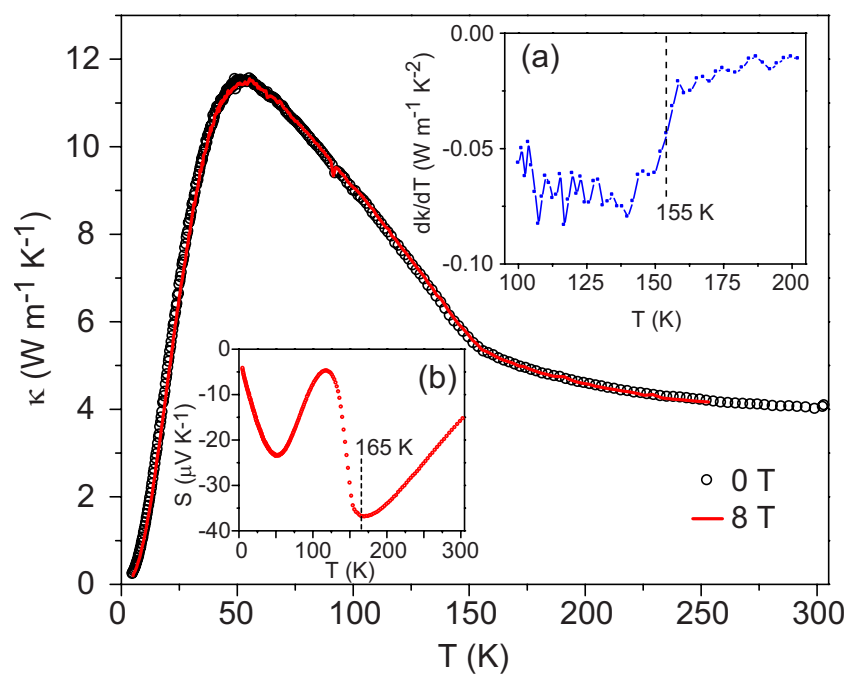

FIG. 8. (Color online) Thermal and thermoelectric transport properties of $\mathrm{LaFeAsO}$. The thermal conductivity measured in zero applied magnetic field and in a field of $8 \mathrm{~T}$, showing an abrupt change in slope [inset (a)] as the structure transforms from tetragonal to orthorhombic upon cooling. Inset (b) shows the dramatic changes in the Seebeck coefficient that occur below the transition temperatures.

sented above [Fig. 7(b)]. An increase in phonon thermal conductivity could also arise from the freezing in of phononscattering lattice fluctuations upon cooling through $\mathrm{T}_{T-O}$.

The Seebeck coefficient (Fig. 8, inset b) is negative over the entire temperature range, indicating that electrons dominate the electrical conduction. This is consistent with the negative Hall coefficient (Fig. 7). The Seebeck coefficient is moderately high in this material, but lower by about a factor of 2 than in superconducting $\mathrm{LaFeAsO}_{0.89} \mathrm{~F}_{0.11}$ above $T_{C}{ }^{8}{ }^{8} \mathrm{~A}$ remarkable decrease in $|S|$ is observed below about $155 \mathrm{~K}$. It is unusual to see a sharp drop in $|S|$ coinciding with a sharp drop in carrier concentration. Boltzmann transport theory predicts for the free-electron model ${ }^{37}$

$$
S(T)=-\frac{\pi^{2}}{3} \frac{k_{B}}{|e|} k_{B} T\left[\frac{N\left(\epsilon_{F}\right)}{n}+\frac{1}{\tau\left(\epsilon_{F}\right)} \frac{d \tau}{d \epsilon_{F}}\right] .
$$

The observed decrease in both $|S|$ and $n$ through the transition indicates that the second term in Eq. (1) is dominant in this temperature regime. This suggests that the charge-carrier scattering mechanism is changed significantly as the material passes through the phase-transition region, and presents further evidence for the reduction in electron-phonon interactions in the orthorhombic phase which was suggested by the above analysis of carrier mobility and thermal conductivity. This is evidence of strong electron-phonon coupling in LaFeAsO.

\section{F. Magnetic properties}

Results of magnetization measurements on a PXRD pure polycrystalline sample of $\mathrm{LaFeAsO}$ (synthesis route $\mathrm{C}$ ) are shown in Fig. 9. Measured $M(H)$ curves (Fig. 9 inset) show a paramagnetic response up to $H=6 \mathrm{~T}$ at 100,150 , and 200

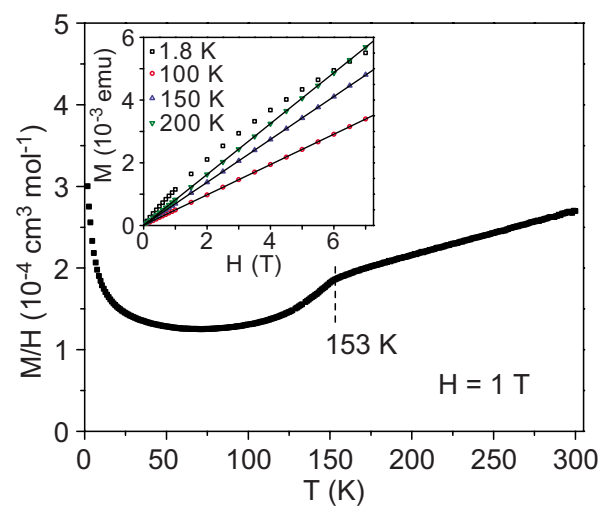

FIG. 9. (Color online) The temperature dependence of $M / H$ (per mole of formula units) for a PXRD pure polycrystalline LaFeAsO sample. The inset shows $M(H)$ data at four different temperatures.

$\mathrm{K}$. The upturn in $M / H$ at low temperatures and the nonlinear behavior of $M(H)$ at $1.8 \mathrm{~K}$ are likely due to small amounts of impurities. Other than the Curie tail at low temperatures, the magnetization shows little temperature dependence above and below the transition region. A decrease in $M / H$ is observed through the phase transitions, beginning near $153 \mathrm{~K}$. This has been identified as a signature of spin-density wave formation. Localization of charge carriers which occurs at $\mathrm{T}_{T-O}$ would also result in a decrease in $M / H$ through the reduction in the Pauli paramagnetism; however, one would expect Curie-Weiss behavior below this temperature if that were the complete story. Neutron diffraction results presented above and elsewhere ${ }^{12}$ suggest that an antiferromagnetic ordering develops near the temperature at which $M / H$ decreases in this material. Carrier localization followed closely by antiferromagnetic ordering could be responsible for the behavior observed near $153 \mathrm{~K}$ in Fig. 9. The magnetic behavior of this anisotropic material is certainly complex and not yet well characterized or well understood. Future studies of single crystals with well controlled stoichiometry will be of great importance to the understanding of the magnetic nature of $\mathrm{LaFeAsO}$.

The Mössbauer spectra of LaFeAsO produced by synthesis route B are shown in Fig. 10. The spectra were modeled using a mixture of LaFeAsO and FeAs (vide infra). Between 4.2 and $75 \mathrm{~K}$, the $\mathrm{LaFeAsO}$ Mössbauer spectrum is a simple magnetic sextet. At $4.2 \mathrm{~K}$, the isomer shift and quadrupole shift of $\mathrm{LaFeAsO}$ are 0.576(5) and -0.031(1) $\mathrm{mm} / \mathrm{s}$, respectively, indicating the low-spin nature of the iron(II). The hyperfine field is 5.19(1) $\mathrm{T}$ and the usual conversion factor of $15 \mathrm{~T}$ per $1 \mu_{B}$ yields an estimated Fe moment of ca. $0.35 \mu_{B}$. These hyperfine parameters are in very good agreement with those reported in Refs. 39-41. Note that in Ref. 40 the "LaFeAs" sample was oxidized and that the spectra are actually those of $\mathrm{LaFeAsO}$. A recent theoretical study has also derived $0.25-0.35 \mu_{B}$ in the low-temperature phase by considering coupling between an itinerant band and a more localized band. ${ }^{42}$ It has also been shown that magnetic frustration effects due to competing nearest-neighbor and nextnearest-neighbor interactions can explain the small moment on $\mathrm{Fe}^{43}$ The small, but nonzero, quadrupole shift indicates 


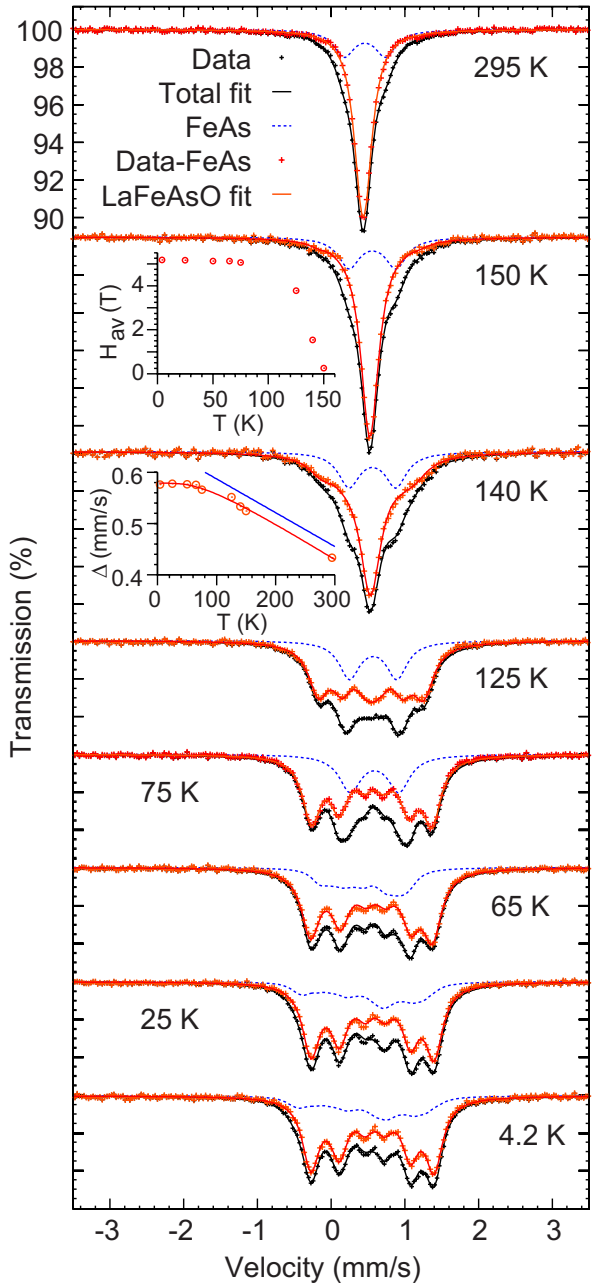

FIG. 10. (Color online) The Mössbauer spectra of LaFeAsO. The total data and fits, as well as the data with the FeAs impurity contribution subtracted and the LaFeAsO phase fit are shown. Upper inset: the average hyperfine field determined in the LaFeAsO phase. Lower inset: The temperature dependence of the isomer shift of LaFeAsO, with a Debye model fit, and, straight line, the isomer shift in FeAs from Ref. 38.

that there is a small lattice contribution to the iron(II) quadrupole interaction, as expected for low-spin iron(II) in a slightly distorted tetrahedral environment. The observed quadrupole shift is constant up to $140 \mathrm{~K}$. At 150 and $295 \mathrm{~K}$ the quadrupole splitting is zero within the error bar. The introduction of a small, temperature independent, texture for the $\mathrm{LaFeAsO}$ phase, with intensity ratios of $3: 2.3: 1: 1: 2.3: 3$ yielded a significant reduction in $\chi^{2}$ and this texture was used for the fits in Fig. 10. The temperature dependence of the isomer shift is in very good agreement with the Debye model for the second-order Doppler shift and yielded a Mössbauer temperature of 294(20) K, see lower inset of Fig. 10. The magnetic moment is essentially constant up to $75 \mathrm{~K}$, and the gradual reduction in the hyperfine field above $125 \mathrm{~K}$ indicates a transition to a paramagnetic phase close $150 \mathrm{~K}$. Between 125 and $150 \mathrm{~K}$, the $\mathrm{LaFeAsO}$ spectrum is more complex, as in Refs. 36 and 39, and was modeled herein as a superposition of a sextet and a singlet, and the weighted average hyperfine field is shown in the upper inset of Fig. 10.
The observed spectra between 125 and $150 \mathrm{~K}$ could be explained either by a small compositional variation and a smearing of $T_{N}$ or by a hyperfine field distribution resulting from a incommensurate or commensurate spin-density wave $^{36,39}$ or from spin-glass behavior in this temperature range. At $75 \mathrm{~K}$ and below, however, the simple sextet spectrum is indicative of no such spin-density wave or spin-glass behavior. Finally, we observe a marginal increase in linewidth on cooling from 295 to $150 \mathrm{~K}$, this increase could be related to the tetragonal to orthorhombic structural distortion.

Because the Mössbauer spectra of $\mathrm{LaFeAsO}$ and FeAs (Refs. 38 and 44) are located in the same velocity region, detecting FeAs impurities requires careful comparison of the spectra near the $77 \mathrm{~K} \mathrm{Neel} \mathrm{temperature} \mathrm{of} \mathrm{FeAs.} \mathrm{It} \mathrm{is} \mathrm{clear}$ that the transition of FeAs from the helimagnetic to paramagnetic phase is responsible for the modification of the spectra between 65 and $75 \mathrm{~K}$. The FeAs contribution above and below $70 \mathrm{~K}$ was found to be consistent with and then constrained to the hyperfine parameters for the corresponding temperatures of Refs. 38 and 44, respectively, and resulting fits are of very good quality. The FeAs contribution corresponds to ca. $10 \%$ weight of the sample, a quantity that is essentially temperature independent, indicating a similar temperature dependence of the recoil free fraction in FeAs and $\mathrm{LaFeAsO}$ and indeed the ca. $285 \mathrm{~K}$ Mössbauer temperature obtained from the second-order Doppler shift in FeAs, Ref. 38 and the line in the lower inset of Fig. 10, is very close to $294 \mathrm{~K}$ observed herein for LaFeAsO. The only free fit parameters for the FeAs phase are the linewidth, and the isomer shift and average hyperfine field at 25,50 , and $65 \mathrm{~K}$. We believe that the larger intensity of the $2: 5$ lines in the spectra of Refs. 36, 39, and 41 arises not from a possible texturing of the sample but from an underlying FeAs impurity subspectrum. The presence of this FeAs impurity, if not properly modeled will lead to a smaller effective hyperfine field, as seen in Ref. 36. Further, it is likely that the third component observed in $\mu S R$ measurements below $70 \mathrm{~K}$ (Ref. 36) is also related to the FeAs impurity that is clearly visible in the $78 \mathrm{~K}$ Mössbauer spectrum on the same sample.

\section{CONCLUSIONS}

At high temperatures $\mathrm{LaFeAsO}$ is a low carrierconcentration metal with conduction dominated by electrons and with no local magnetic moment. This is supported by measurements of electrical resistivity (Fig. 6), Hall coefficient and carrier concentration (Fig. 7), Seebeck coefficient (Fig. 8), and magnetization (Fig. 9). Upon cooling a crystallographic phase transition occurs. We propose that the structural transformation occurs continuously below about $200 \mathrm{~K}$ based on structure refinements (Fig. 2) and elastic response (Fig. 4), and that the kink in the order parameter $b$ - $a$ at 160 $\mathrm{K}$ [Fig. 2(c)] indicates the point at which sufficient distortion has occurred to result in carrier localization and localmoment formation on the Fe atoms. This is supported by the small entropy associated with the transition at $\mathrm{T}_{T-O}$ (Fig. 5). It is unclear what drives this structural transition. One candidate is a band Jahn-Teller effect, ${ }^{45}$ in which energy is gained by splitting the sharp peak in the density of states 
near the Fermi level ${ }^{10}$ by distorting the crystal structure. This could result in localization of some conduction electrons and the development of a local magnetic moment on Fe. Careful theoretical analysis of the effects of structural distortion on the band structure of $\mathrm{LaFeAsO}$ could help resolve this issue. Upon further cooling the local moments order near $145 \mathrm{~K}$ (Fig. 3). Strong electron-phonon coupling exists in the hightemperature tetragonal phase, as evidenced by the behavior of the mobility [Fig. 7(b)], thermal conductivity, and Seebeck coefficient (Fig. 8) through the phase-transition region. Upon doping with, for example, fluorine, the structural phase transformation and associated magnetic ordering is suppressed (Fig. 3), allowing the strong electron-phonon coupling present in the tetragonal phase to extend down to low temperature, and superconductivity emerges. It is believed that the phase transition is suppressed electronically by electron or hole doping with substitutional F or Sr, or with oxygen vacancies. However, disorder may also suppress the structural transition. Perhaps other superconductors can be discovered by investigating isoelectronic substitutions which suppress $\mathrm{T}_{T-O}$ in the $\mathrm{ZrCuSiAs-type} \mathrm{rare-earth}$ iron oxyars- enides and related $\mathrm{ThCr}_{2} \mathrm{Si}_{2}$-type alkaline-earth iron arsenides.

\section{ACKNOWLEDGMENTS}

We acknowledge useful discussions with D. J. Singh, O. Garlea, S. Nagler, A. Castro-Neto, and I. I. Mazin, and technical assistance by J. Zarestky and J. Fernandez-Baca. Research sponsored by the Division of Materials Sciences and Engineering, Office of Basic Energy Sciences. Part of this research performed by Eugene P. Wigner Fellows at ORNL, managed by UT-Battelle, LLC, for the U.S. DOE under Contract No. DE-AC05-00OR22725. A portion of this research at ORNL's Center for Nanophase Materials Sciences and High Flux Isotope Reactor was sponsored by the Scientific User FacilitiesDivision, Office of Basic Energy Sciences, DOE. A portion of this work was supported by NSF Grant No. DMR-0400938. F.G. acknowledges with thanks the financial support of the Fonds National de la Recherche Scientifique, Belgium, through Grants No. 9.456595 and No. 1.5.064.05.
${ }^{1}$ P. Quebe, L. J. Terbüe, and W. Jeitschko, J. Alloys Compd. 302, 70 (2000).

${ }^{2}$ B. Zimmer, W. Jeitschko, J. H. Albering, R. Glaum, and M. Reehuis, J. Alloys Compd. 229, 238 (1995).

${ }^{3}$ Y. Kamihara, H. Hiramatsu, M. Hirano, R. Kawamura, H. Yanagi, T. Kamiya, and H. Hosono, J. Am. Chem. Soc. 128, 10012 (2006).

${ }^{4}$ C. Y. Liang, R. C. Che, H. X. Yang, H. F. Tian, R. J. Xiao, J. B. Lu, R. Li, and J. Q. Li, Supercond. Sci. Technol. 20, 687 (2007).

${ }^{5}$ T. Watanabe, H. Yanagi, T. Kamiya, Y. Kamihara, H. Hiramatsu, M. Hirano, and H. Hosono, Inorg. Chem. 46, 7719 (2007).

${ }^{6}$ Y. Kamihara, T. Watanabe, M. Hirano, and H. Hosono, J. Am. Chem. Soc. 130, 3296 (2008).

${ }^{7}$ F. Hunte, J. Jaroszynski, A. Gurevich, D. C. Larbalestier, R. Jin, A. S. Sefat, M. A. McGuire, B. C. Sales, D. K. Christen, and D. Mandrus, Nature (London) 453, 903 (2008).

${ }^{8}$ A. S. Sefat, M. A. McGuire, B. C. Sales, R. Jin, J. Y. Howe, and D. Mandrus, Phys. Rev. B 77, 174503 (2008).

${ }^{9}$ X. H. Chen, T. Wu, G. Wu, R. H. Liu, H. Chen, and D. F. Fang, Nature (London) 453, 761 (2008).

${ }^{10}$ D. J. Singh and M. H. Du, Phys. Rev. Lett. 100, 237003 (2008).

${ }^{11}$ Y. Sun, M. Guidry, and C. Wu, Chin. Sci. Bull. 53, 1617 (2008).

${ }^{12}$ C. de la Cruz et al., Nature (London) 453, 899 (2008).

${ }^{13}$ D. Wu, Z. Li, S. Su, J. Dong, G. Li, W.-Z. Hu, P. Zheng, G.-F. Chen, J.-L. Luo, and N.-L. Wang, Sci. China, Ser. G 51, 715 (2008).

${ }^{14}$ P. Cheng, L. Fang, H. Yang, X.-Y. Zhu, G. Mu, H.-Q. Luo, Z.-S. Wang, and H.-H. Wen, Sci. China, Ser. G 51, 719 (2008).

${ }^{15}$ S. Ishibashi, K. Terakura, and H. Hosono, J. Phys. Soc. Jpn. 77, 053709 (2008).

${ }^{16}$ C. Day, Phys. Today 61 (5), 11 (2008).

${ }^{17}$ H. Takahashi, K. Igawa, K. Arii, Y. Kamihara, M. Hirano, and H. Hosono, Nature (London) 453, 376 (2008).

${ }^{18}$ Q. Han, Y. Chen, and Z. D. Wang, EPL 82, 37007 (2008).
${ }^{19}$ K. Haule, J. H. Shim, and G. Kotliar, Phys. Rev. Lett. 100, 226402 (2008).

${ }^{20}$ H.-H. Wen, G. Mu, L. Fang, H. Yang, and X. Zhu, EPL 82, 17009 (2008).

${ }^{21}$ E. Hand, Nature (London) 452, 922 (2008).

${ }^{22}$ H. Kito, H. Eisaki, and A. Iyo, J. Phys. Soc. Jpn. 77, 063707 (2008).

${ }^{23}$ T. Sato, S. Souma, K. Nakayama, K. Terashima, K. Sugawara, T. Takahashi, Y. Kamihara, M. Hirano, and H. Hosono, J. Phys. Soc. Jpn. 77, 063708 (2008).

${ }^{24}$ T. Y. Chen, Z. Tesanovic, R. H. Liu, X. H. Chen, and C. L. Chien, Nature (London) 453, 1224 (2008)

${ }^{25}$ Z.-A. Ren et al., EPL 82, 57002 (2008).

${ }^{26}$ Z.-A. Ren, J. Yang, W. Lu, W. Yi, G.-C. Che, X.-L. Dong, L.-L. Sun, and Z.-X. Zhao, EPL 83, 17002 (2008).

${ }^{27}$ G. Xu, W. Ming, Y. Yao, X. Dai, S.-C. Zhang, and Z. Fang, EPL 82, 67002 (2008).

${ }^{28}$ I. I. Mazin, D. J. Singh, M. D. Johannes, and M. H. Du, Phys. Rev. Lett. 101, 057003 (2008).

${ }^{29}$ J. Dong et al., EPL 83, 27006 (2008).

${ }^{30}$ T. Nomura, S. W. Kim, Y. Kamihara, M. Hirano, P. V. Sushko, K. Kato, M. Takata, A. L. Shluger, and H. Hosono, arXiv:0804.3569 (unpublished).

${ }^{31}$ J. Rodriguez-Carvajal, FULLPROF Suite 2005, Version 3.30, June 2005.

${ }^{32}$ B. H. Toby, J. Appl. Crystallogr. 34, 210 (2001).

${ }^{33}$ A. C. Larson and R. B. V. Dreele, "General structural analysis system (GSAS)," Los Alamos National Laboratory Report No. LAUR 86-748, 2004.

${ }^{34}$ M. Chung, E. Figueroa, Y.-K. Kuo, Y. Wang, J. W. Brill, T. Burgin, and L. K. Montgomery, Phys. Rev. B 48, 9256 (1993).

${ }^{35}$ A. Migliori and J. Maynard, Rev. Sci. Instrum. 76, 121301 (2005).

${ }^{36}$ H.-H. Klauss et al., Phys. Rev. Lett. 101, 077005 (2008). 
${ }^{37}$ J. McCarten, S. E. Brown, C. L. Seaman, and M. B. Maple, Phys. Rev. B 49, 6400 (1994).

${ }^{38}$ S. Kulshreshtha and P. Raj, J. Phys. F: Met. Phys. 9, 2253 (1979).

${ }^{39}$ S. Kitao, Y. Kobayashi, S. Higashitaniguchi, M. Saito, Y. Kamihara, M. Hirano, T. Mitsui, H. Hosono, and M. Seto, arXiv:0805.0041 (unpublished).

${ }^{40}$ H. Raffius, E. Mörsen, B. Mosel, W. Müller-Warmuth, W. Jeitschko, L. Terbüchte, and T. Vomhof, J. Phys. Chem. Solids
54, 135 (1993).

${ }^{41}$ I. Nowik, I. Felner, V. Awana, A. Vajpayee, and H. Kishan, J. Phys.: Condens. Matter 20, 292201 (2008).

${ }^{42}$ J. Wu, P. Phillips, and A. H. Castro Neto, Phys. Rev. Lett. 101, 126401 (2008).

${ }^{43}$ Q. Si and E. Abrahams, Phys. Rev. Lett. 101, 076401 (2008).

${ }^{44}$ L. Häggström, A. Gustavsson-Seidel, and H. Fjellvåg, Europhys. Lett. 9, 87 (1989).

${ }^{45}$ R. N. Bhatt, Phys. Rev. B 16, 1915 (1977). 